\title{
Modulus of Convexity, the Coeffcient $R(1, X)$, and Normal Structure in Banach Spaces
}

\author{
Hongwei Jiao, ${ }^{1}$ Yunrui Guo, ${ }^{1}$ and Fenghui Wang ${ }^{2}$ \\ ${ }^{1}$ Department of Mathematics, Henan Institute of Science and Technology, Xinxiang 453003, China \\ ${ }^{2}$ Department of Mathematics, Luoyang Normal University, Luoyang 471022, China
}

Correspondence should be addressed to Hongwei Jiao, jiaohongwei@126.com

Received 7 April 2008; Accepted 27 May 2008

Recommended by William A. Kirk

Let $\delta_{X}(\epsilon)$ and $R(1, X)$ be the modulus of convexity and the Domínguez-Benavides coefficient, respectively. According to these two geometric parameters, we obtain a sufficient condition for normal structure, that is, a Banach space $X$ has normal structure if $2 \delta_{X}(1+\epsilon)>\max \{(R(1, x)-$ 1) $\epsilon, 1-(1-\epsilon / R(1, X)-1)\}$ for some $\epsilon \in[0,1]$ which generalizes the known result by Gao and Prus.

Copyright ( $\odot 2008$ Hongwei Jiao et al. This is an open access article distributed under the Creative Commons Attribution License, which permits unrestricted use, distribution, and reproduction in any medium, provided the original work is properly cited.

\section{Introduction}

Let $X$ be a Banach space. Throughout the paper, denote by $S_{X}, B_{X}$ the unit sphere and unit ball of $X$, respectively. Recall that a Banach space $X$ is said to have normal structure (resp., weak, normal, structure) if for every closed bounded (resp., weakly compact) convex subset $C$ of $X$ with diam $C>0$, there exists $x \in C$ such that $\sup \{\|x-y\|: y \in C\}<\operatorname{diam} C$, where diam $C=\sup \{\|x-y\|: x, y \in C\}$. For a reflexive Banach space, the normal structure and weak normal structure are the same. Recently a good deal of investigations have focused on finding the sufficient conditions with various geometrical constants for a Banach space to have normal structure (see, e.g., [1-5]). The geometric condition sufficient for normal structure in terms of the modulus of convexity is given by Goebel [6], who proved that $X$ has normal structure provided that $\delta_{X}(1)>0$. Here the function $\delta_{X}(\epsilon):[0,2] \rightarrow[0,1]$, defined by Clarkson [7] as

$$
\delta_{X}(\epsilon)=\inf \left\{1-\frac{\|x+y\|}{2}: x, y \in B_{X},\|x-y\| \geq \epsilon\right\},
$$

is called the modulus of convexity of $X$. Later Gao and Prus generalized the above results as the following (see $[2,8]$ ). 
Theorem 1.1. A Banach space $X$ has normal structure provided that $\delta_{X}(1+\epsilon)>\epsilon / 2$ for some $\epsilon \in$ $[0,1]$.

In this paper, we obtain a class of Banach spaces with normal structure, which involves the coefficient $R(1, X)$. This coefficient is defined by Domínguez Benavides [9] as

$$
R(1, X)=\sup \left\{\liminf _{n \rightarrow \infty}\left\|x+x_{n}\right\|\right\},
$$

where the supremum is taken over all $x \in X$ with $\|x\| \leq 1$ and all weakly null sequence $\left(x_{n}\right)$ in $B_{X}$ such that

$$
D\left[\left(x_{n}\right)\right]:=\limsup _{n \rightarrow \infty}\left(\limsup _{m \rightarrow \infty}\left\|x_{n}-x_{m}\right\|\right) \leq 1 .
$$

Obviously, $1 \leq R(1, X) \leq 2$.

\section{Main results}

Let us begin this section with a sufficient condition for a Banach space $X$ having weak normal structure and the idea in the following proof is due to [5, Lemma 5].

Lemma 2.1. Let $X$ be a Banach space for which $B_{X^{*}}$ is $w^{*}$-sequentially compact. If $X$ does not have weak normal structure, then for any $\eta>0$, there exist $x_{1}, x_{2} \in S_{X}$ and $f_{1}, f_{2} \in S_{X^{*}}$, such that

(1) ||$\left|x_{1}-x_{2} \|-1\right|<\eta$;

(2) $\left|f_{i}\left(x_{j}\right)\right|<\eta$ for $i \neq j$ and $f_{i}\left(x_{i}\right)=1, i, j=1,2$;

(3) $\left\|x_{1}+x_{2}\right\| \leq R(1, X)(1+\eta)$.

Proof. Assume that $X$ does not have weak normal structure. It is well known that (see, e.g., [10]) there exists a sequence $\left\{x_{n}\right\}$ in $X$ satisfying

(1) $x_{n}$ is weakly convergent to 0 ;

(2) $\operatorname{diam}\left(\left\{x_{n}\right\}_{n=1}^{\infty}\right)=1=\lim _{n}\left\|x_{n}-x\right\|$ for all $x \in \operatorname{clco}\left\{x_{n}\right\}_{n=1}^{\infty}$.

Since $B_{X^{*}}$ is $w^{*}$-sequentially compact, we can find $\left\{f_{n}\right\}$ in $S_{X^{*}}$ satisfying

(3) $f_{n}\left(x_{n}\right)=\left\|x_{n}\right\|$ for all $n$;

(4) $f_{n} \stackrel{w^{*}}{\rightarrow} f$ for some $f \in B_{X^{*}}$.

Let $\eta \in(0,1)$ sufficiently small and $\epsilon=\eta / 3$. Then, by the properties of the sequence $\left(x_{n}\right)$, we can choose $n_{1} \in \mathbb{N}$ such that

$$
\left|f\left(x_{n_{1}}\right)\right|<\frac{\epsilon}{2}, \quad 1-\epsilon \leq\left\|x_{n_{1}}\right\| \leq 1 .
$$

Note that the sequence $\left\{x_{n}\right\}$ is weakly null and verifies $D\left[\left\{x_{n}\right\}\right]=1$. It follows from the definition of $R(1, X)$ that

$$
\liminf _{n}\left\|x_{n}+x_{n_{1}}\right\| \leq R(1, X) .
$$

The rest of the proof is similar to that of [5, Lemma 5]. 
Hongwei Jiao et al.

Theorem 2.2. A Banach space $X$ has normal structure provided that $\delta_{X}(1+\epsilon)>f(\epsilon)$ for some $\epsilon \in$ $[0,1]$, where the function $f(\epsilon)$ is defined as

$$
f(\epsilon):= \begin{cases}(R(1, X)-1) \frac{\epsilon}{2}, & 0 \leq \epsilon \leq \frac{1}{R(1, X)}, \\ \frac{1}{2}\left(1-\frac{1-\epsilon}{R(1, X)-1}\right), & \frac{1}{R(1, X)}<\epsilon \leq 1 .\end{cases}
$$

Proof. Observe that $X$ is uniformly nonsquare [11] and then reflexive. Therefore normal structure and weak normal structure coincide.

Assume first that $X$ fails to have weak normal structure. Fix $\eta>0$ sufficiently small and $\epsilon \in[0,1]$. It follows that there exist $x_{1}, x_{2} \in S_{X}$ and $f_{1}, f_{2} \in S_{X^{*}}$, satisfying the condition in Lemma 2.1. Next, denote by $R:=R(1, X)$ and consider two cases for $\epsilon \in[0,1]$.

Case $1(\epsilon \in[0,1 / R])$. Now let us put

$$
x=\frac{x_{1}-x_{2}}{1+\eta}, \quad y=\frac{(1-(R-1) \epsilon) x_{1}+\epsilon x_{2}}{1+\eta},
$$

and so $x \in B_{X}$,

$$
\|y\|=\left\|\frac{\epsilon}{1+\eta}\left(x_{1}+x_{2}\right)+\frac{1-R \epsilon}{1+\eta} x_{1}\right\| \leq R \epsilon+(1-R \epsilon)=1
$$

and also that

$$
\begin{aligned}
\|x-y\| & =\left\|\frac{(R-1) \epsilon}{1+\eta} x_{1}-\frac{1+\epsilon}{1+\eta} x_{2}\right\| \geq \frac{1+\epsilon}{1+\eta} f_{2}\left(x_{2}\right)-\frac{(R-1) \epsilon}{1+\eta} f_{2}\left(x_{1}\right) \geq \frac{1+\epsilon-\eta}{1+\eta} \\
\|x+y\| & =\left\|\frac{(2-(R-1)) \epsilon}{1+\eta} x_{1}-\frac{1-\epsilon}{1+\eta} x_{2}\right\| \geq \frac{(2-(R-1)) \epsilon}{1+\eta} f_{1}\left(x_{1}\right)-\frac{1-\epsilon}{1+\eta} f_{1}\left(x_{2}\right) \\
& \geq\left(1-\frac{2 \eta}{1+\eta}\right)(2-(R-1) \epsilon) .
\end{aligned}
$$

By the definition of modulus of convexity,

$$
\left(1-\frac{2 \eta}{1+\eta}\right)(2-(R-1) \epsilon) \leq\|x+y\| \leq 2\left(1-\delta_{X}(\|x-y\|)\right) \leq 2\left(1-\delta_{X}\left(\frac{1+\epsilon-\eta}{1+\eta}\right)\right)
$$

or equivalently,

$$
(2-(R-1) \epsilon) \leq 2\left(1-\delta_{X}\left(\frac{1+\epsilon-\eta}{1+\eta}\right)\right)\left(1+\frac{2 \eta}{1-\eta}\right) .
$$

Letting $\eta \rightarrow 0$, we have

$$
2 \delta_{X}(1+\epsilon) \leq(R-1) \epsilon,
$$

which contradicts our hypothesis. 
Case $2(\epsilon \in(1 / R, 1])$. In this case $R>1$, otherwise $\epsilon>1$. Let

$$
x^{\prime}=\frac{x_{2}-x_{1}}{1+\eta}, \quad y^{\prime}=\frac{\left(1-(R-1) \epsilon^{\prime}\right) x_{1}+\epsilon^{\prime} x_{2}}{1+\eta}
$$

where $\epsilon^{\prime}=1-(R-1) \epsilon \in[0,1 / R)$. It follows from Case 1 that $x, y \in B_{X}$,

$$
\|x-y\| \geq\left(1-\frac{2 \eta}{1+\eta}\right)\left(2-(R-1) \epsilon^{\prime}\right), \quad\|x+y\| \geq \frac{1+\epsilon^{\prime}-\eta}{1+\eta}
$$

This implies that

$$
\delta_{X}\left(2-(R-1) \epsilon^{\prime}\right) \leq \frac{1}{2}\left(1-\epsilon^{\prime}\right)
$$

which is equivalent to

$$
\delta_{X}(1+\epsilon) \leq \frac{1}{2}\left(1-\frac{1-\epsilon}{R-1}\right)
$$

This is a contradiction.

Remark 2.3. (1) It is readily seen that $f(\epsilon) \leq \epsilon / 2$ for any $\epsilon \in[0,1]$ and Theorem 2.2 is therefore a generalization of Theorem 1.1. Moreover this generalization is strict whenever $X$ is the space with $R(1, X)<2$.

(2) Consider the space $X=\mathbb{R}^{2}$ with the norm $\|(x, y)\|:=\max (|x|,|y|,|x-y|)$. It is known that $\delta_{X}(\epsilon)=\max \{0,(\epsilon-1) / 2\}$ [8] and $R(1, X)=1$, then $X$ has normal structure from Theorem 2.2, but lies out of the scope of Theorem 1.1 .

Corollary 2.4. Let $X$ be a Banach space with $R(1, X)=1$ and $\delta_{X}(1+\epsilon)>0$ for some $\epsilon \in[0,1]$, then $X$ has normal structure.

Corollary 2.5. If $X$ is a Banach space with

$$
\delta_{X}\left(1+\frac{1}{R(1, X)}\right)>\frac{1}{2}\left(1-\frac{1}{R(1, X)}\right)
$$

then $\mathrm{X}$ has normal structure.

Remark 2.6. Corollary 2.5 is equivalent to [4, Corollary 24].

\section{Acknowledgments}

The authors would like to express their sincere thanks to the referees for their valuable suggestions on this paper. This work is supported by NSF of Education Department of Henan Province (2008A110012). 


\section{References}

[1] S. Dhompongsa, A. Kaewkhao, and S. Tasena, "On a generalized James constant," Journal of Mathematical Analysis and Applications, vol. 285, no. 2, pp. 419-435, 2003.

[2] J. Gao, "Modulus of convexity in Banach spaces," Applied Mathematics Letters, vol. 16, no. 3, pp. 273278, 2003.

[3] A. Jiménez-Melado, E. Llorens-Fuster, and S. Saejung, "The von Neumann-Jordan constant, weak orthogonality and normal structure in Banach spaces," Proceedings of the American Mathematical Society, vol. 134, no. 2, pp. 355-364, 2006.

[4] E. M. Mazcuñán-Navarro, "Banach space properties sufficient for normal structure," Journal of Mathematical Analysis and Applications, vol. 337, no. 1, pp. 197-218, 2008.

[5] S. Saejung, "The characteristic of convexity of a Banach space and normal structure," Journal of Mathematical Analysis and Applications, vol. 337, no. 1, pp. 123-129, 2008.

[6] K. Goebel, "Convexivity of balls and fixed-point theorems for mappings with nonexpansive square," Compositio Mathematica, vol. 22, pp. 269-274, 1970.

[7] J. A. Clarkson, "Uniformly convex spaces," Transactions of the American Mathematical Society, vol. 40, no. 3, pp. 396-414, 1936.

[8] S. Prus, "Some estimates for the normal structure coefficient in Banach spaces," Rendiconti del Circolo Matematico di Palermo, vol. 40, no. 1, pp. 128-135, 1991.

[9] T. Domínguez Benavides, "A geometrical coefficient implying the fixed point property and stability results," Houston Journal of Mathematics, vol. 22, no. 4, pp. 835-849, 1996.

[10] K. Goebel and W. A. Kirk, Topics in Metric Fixed Point Theory, vol. 28 of Cambridge Studies in Advanced Mathematics, Cambridge University Press, Cambridge, UK, 1990.

[11] M. Kato, L. Maligranda, and Y. Takahashi, "On James and Jordan-von Neumann constants and the normal structure coefficient of Banach spaces," Studia Mathematica, vol. 144, no. 3, pp. 275-295, 2001. 


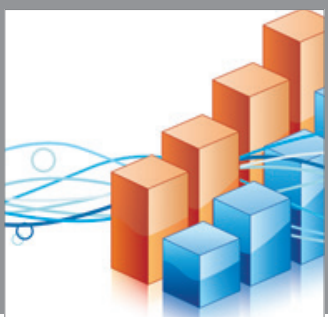

Advances in

Operations Research

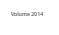

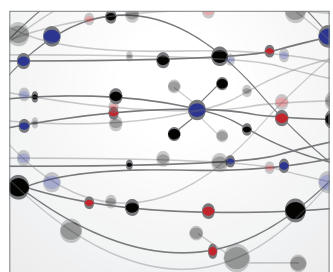

\section{The Scientific} World Journal
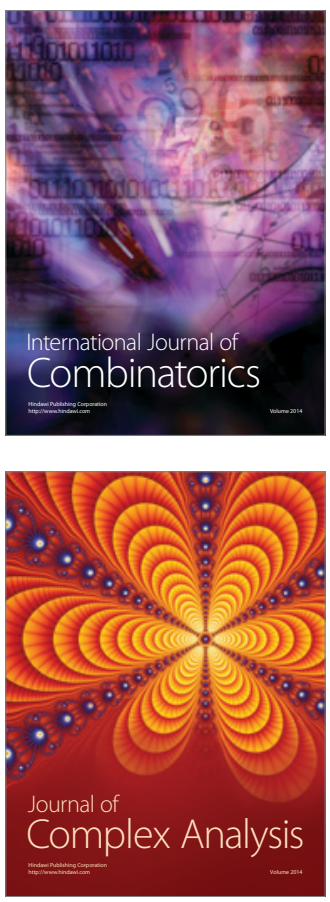

International Journal of

Mathematics and

Mathematical

Sciences
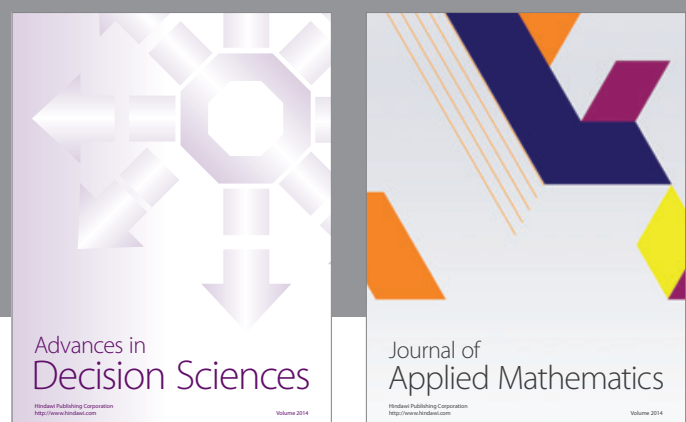

Journal of

Applied Mathematics
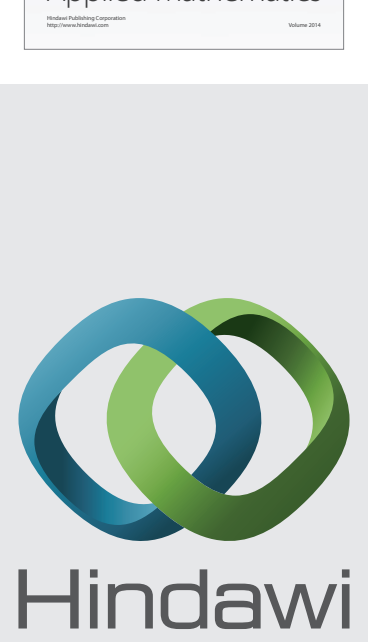

Submit your manuscripts at http://www.hindawi.com
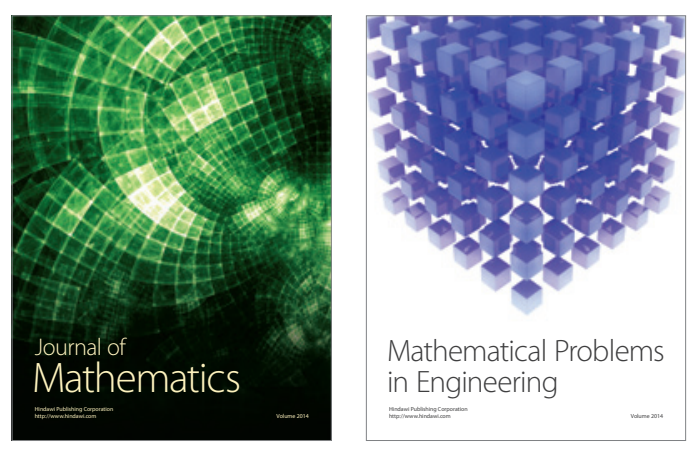

Mathematical Problems in Engineering
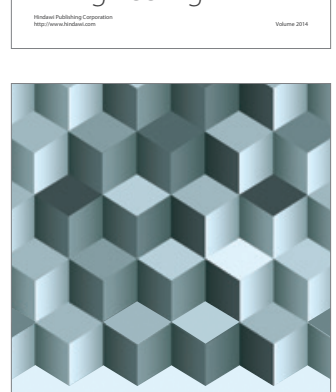

Journal of

Function Spaces
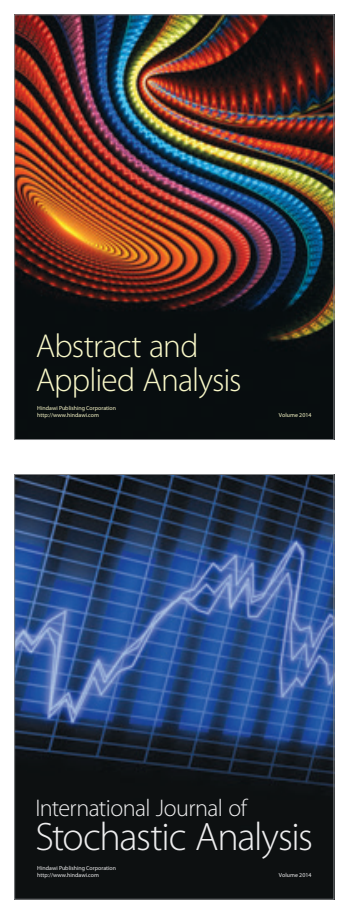

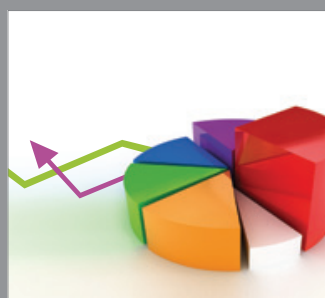

ournal of

Probability and Statistics

Promensencen
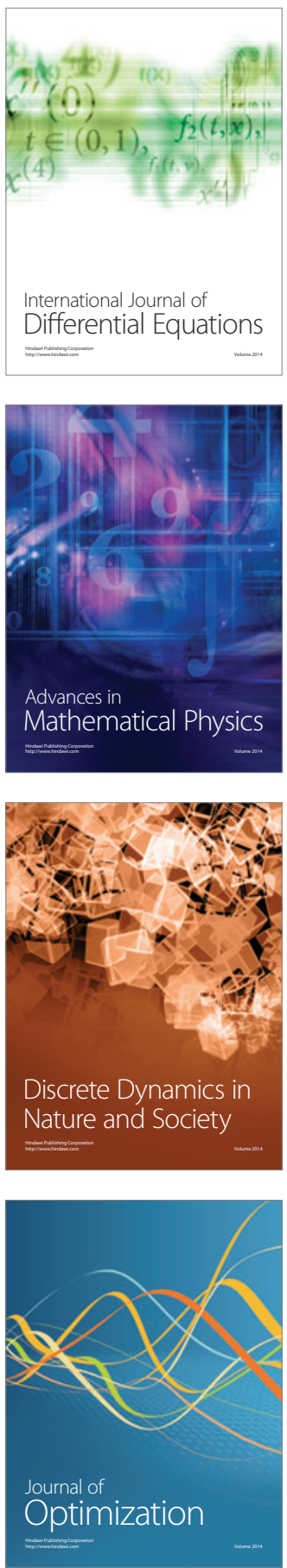